\title{
Inverse modelling of an aneurysm's stiffness using surrogate-based optimization and fluid-structure interaction simulations
}

\author{
Joris Degroote · Ivo Couckuyt • Jan \\ Vierendeels · Patrick Segers • Tom \\ Dhaene
}

Received: date / Accepted: date

\begin{abstract}
Characterization of the mechanical properties of arterial tissues is highly relevant. In this work, we apply an inverse modelling approach to a model accounting for an aneurysm and the distal part of the circulation which can be modified using two independent stiffness parameters. For given values of these parameters, the position of the arterial wall as a function of time is calculated using a forward simulation which takes the fluid-structure interaction (FSI) into account. Using this forward simulation, the correct values of the stiffness parameters are obtained by minimizing a cost function, which is defined as the difference between the forward simulation and a measurement. The
\end{abstract}

\author{
J. Degroote \\ Ghent University, Department of Flow, Heat and Combustion Mechanics \\ Sint-Pietersnieuwstraat 41, B-9000 Ghent, Belgium \\ E-mail: Joris.Degroote@UGent.be \\ I. Couckuyt \\ Ghent University - IBBT, Department of Information Technology, \\ Internet Based Communication Networks and Services (IBCN) \\ Gaston Crommenlaan 8 Bus 201, B-9050 Ghent, Belgium \\ E-mail: Ivo.Couckuyt@UGent.be \\ J. Vierendeels \\ Ghent University, Department of Flow, Heat and Combustion Mechanics \\ Sint-Pietersnieuwstraat 41, B-9000 Ghent, Belgium \\ E-mail: Jan.Vierendeels@UGent.be \\ P. Segers \\ Ghent University, Department of Civil Engineering, \\ Institute Biomedical Technology \\ De Pintelaan 185, B-9000 Ghent, Belgium \\ E-mail: Patrick.Segers@UGent.be \\ T. Dhaene \\ Ghent University - IBBT, Department of Information Technology, \\ Internet Based Communication Networks and Services (IBCN) \\ Gaston Crommenlaan 8 Bus 201, B-9050 Ghent, Belgium \\ E-mail: Tom.Dhaene@UGent.be
}


minimization is performed by means of surrogate-based optimization using a Kriging model combined with the expected improvement infill criterion. The results show that the stiffness parameters converge to the correct values, both for a zero-dimensional and for a three-dimensional model of the aneurysm.

Keywords Fluid-structure interaction · Aneurysm · Parameter identification · Inverse problem · Surrogate model $\cdot$ Expected improvement

\section{Introduction}

The arterial system is a large and complex three-dimensional network playing a vital role in human well-being. Recent advances in the field of numerical analysis and the increase in computer power enable detailed, three-dimensional (3D) fluid-structure interaction (FSI) simulations of, for example, heart valves [2] and aneurysms in large arteries $[13,19]$. In these FSI simulations, the interaction between the blood flow and the surrounding tissue is taken into account. However, patient-specific data such as the initial shape, initial stresses, boundary conditions and constitutive data for the tissue are required as input for these simulations. Non-invasive imaging techniques can provide geometrical data but it is difficult to directly determine the stiffness parameters of the tissue. This is of particular interest within the context of (aortic) aneurysms, where modelling simulations are thought to contribute to a better assessment of the aneurysm's risk of rupture.

The goal of this work is to test the feasibility of assessing the stiffness parameters of an aneurysm in a large artery using inverse modelling. To that end, two stiffness parameters are defined: one for the aneurysm itself and one for the distal part of the arterial system. For given values of these parameters, a forward simulation of the arterial system can be performed, resulting in the position of the arterial wall as a function of time, information that is easily available from medical images. A cost function is then defined to quantify the difference between the wall position in this simulation and in a measurement. Ideally, this measurement should originate from non-invasive imaging techniques but in this work synthetic data from a simulation is used to provide the proof of principle. Future work will discuss the difficulties due to the noise in real measurement data. Yet, the parameter identification technique used in this work is expected to function well in that case [31]. The values of the stiffness parameters that minimize the cost function are then determined by means of surrogate-based optimization [15] (SBO).

Previous parameter identification studies on arteries used one-dimensional models and adjoint techniques for the optimization [20,21]. In this work, the physics of the 3D aneurysm model are fully nonlinear. The geometry of the model is however simplified and not patient-specific. The fluid-structure interaction is simulated by coupling $[9,11]$ a black-box computational fluid dynamics (CFD) solver with a black-box computational structural dynamics (CSD) solver. Consequently, adjoint techniques are not applicable. 
The remainder of this work is organized as follows. Section 2 describes the surrogate-based optimization technique. In Section 3, the models for the forward simulation are introduced, followed by the results in Section 4. Finally, Section 5 presents the conclusions.

\section{Surrogate-based optimization}

Surrogate-based optimization techniques are applied to expedite the optimization of computationally expensive problems [26]. In these optimization techniques, the surrogate model provides a cheap approximation to an expensive calculation with the simulation code. Surrogate models have been incorporated in the optimization process in several ways. They can guide the global search and/or serve as local model for evolutionary optimization algorithms [24,33]. Surrogate models can also be applied in a trust region model-management framework to optimize systems with complex local behaviour [1]. Moreover, models with different levels of accuracy can be combined in multi-fidelity techniques $[16,14,28]$.

Another approach to surrogate-based optimization is applying adaptive sampling strategies, also known as infill criteria, to improve the surrogate models. The infill criterion is a figure of merit that indicates how interesting each point in the design space is. Additional sample points are then selected by optimizing this criterion. The Expected Improvement (EI) infill criterion [18, $23,5]$ effectively balances between enhancing the global accuracy of the surrogate model (exploration) and improving its accuracy near the current optimum (exploitation). Surrogate-based optimization with the expected improvement as infill criterion is also known as the Efficient Global Optimization (EGO) algorithm [18]. This technique requires that the surrogate model provides a Gaussian probability density function (PDF) at each point in the design space. For example, Gaussian Process (GP) surrogate methods provide a normal distribution at each point $x$, determined by the mean $\mu(x)$ and variance $\sigma^{2}(x)$.

The expected improvement infill criterion is explained graphically in Figure 1 . The cost function $f(x)$, which is calculated with the simulation code, has been sampled at 7 points. The value of $f$ at each point $x$ is treated as a random variable $Y(x)$. Assuming that $Y(x)$ is normally distributed with mean $\mu(x)$ and variance $\sigma^{2}(x)$, the probability density function $\phi$ is depicted for $x=1$ and $x=3$. The value predicted by the surrogate model is equal to the mean of this distribution, so $\hat{y}(x)=\mu(x)$. The shaded area corresponds with the Probability of Improvement (PoI) of $Y(x)$ over the current minimum $f_{\text {min }}$, given by

$$
\operatorname{PoI}(x)=P\left(Y(x) \leq f_{\text {min }}\right)=\int_{-\infty}^{f_{\text {min }}} \phi(Y(x)) \mathrm{d} Y
$$

with $\phi$ the normal probability density function. However, this criterion does not take into account how large the improvement will be. Therefore, the Expected Improvement (EI) is the first moment of the shaded area, calculated 


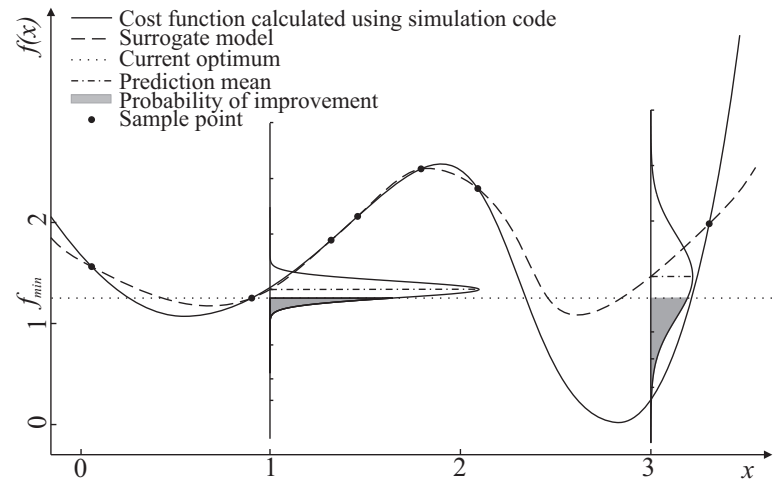

Fig. 1: The cost function $f(x)$, which is calculated using the simulation code, together with a Gaussian process surrogate model. The probability of improvement over the current optimum $f_{\min }$ is shown for $x=1$ and $x=3$.

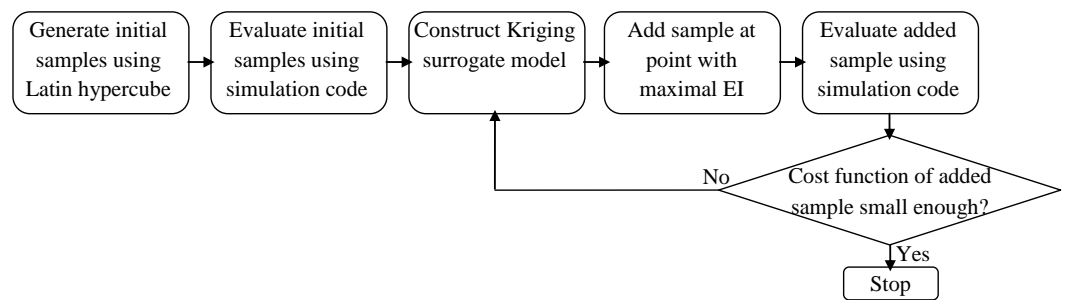

Fig. 2: Schematic of the complete surrogate-based optimization strategy.

as

$$
E I(x)=E[I(x)]=\int_{-\infty}^{f_{m i n}} I(x) \phi(Y(x)) \mathrm{d} Y
$$

for continuous functions with the improvement defined as

$$
I(x)=\max \left(f_{\min }-Y(x), 0\right) .
$$

Equation (2a) corresponds with every possible improvement $I(x)$ over $f_{\text {min }}$, multiplied with the associated likelihood.

In this work, the parameter identification is performed using the SUrrogate MOdelling (SUMO) toolbox [15]. The interested reader can certainly find other packages, e.g. those available at http://www.kernel-machines.org, the free companion code of [12], the SURROGATES Toolbox [32], and the DiceKriging and DiceOptim packages [29]. The complete optimization strategy is summarized in Figure 2. 
Two parameters $\left(x_{1}\right.$ and $\left.x_{2}\right)$ are identified. They can both vary from -1 to 1 , so the parameter space is given by

$$
\left\{\left(x_{1}, x_{2}\right) \mid-1 \leq x_{1} \leq 1,-1 \leq x_{2} \leq 1\right\}
$$

First, an initial set of samples is generated by an optimal maximin Latin hypercube design [7], together with the corner points of the parameter space. Adding the corner points improves the accuracy of the Kriging model in the vicinity of those points. The cost function value $f$ at all initial sample points is evaluated using the simulation code before the first surrogate model is constructed.

The surrogate model of the relation between the stiffness parameters and the cost function is a Kriging model. Kriging is part of the broader class of Gaussian process methods. For a description of Kriging, the reader is referred to the extensive literature on this topic $[30,6]$. The model has a constant regression term and uses the Matérn covariance function with $\nu=3 / 2$ and isotropic distance measure [22]. The Matérn covariance function has been chosen because it yields a smooth surrogate model, whereas surrogate models using several other functions displayed unphysical wiggles. The Kriging model's hyperparameters are determined by Maximum Likelihood Estimation (MLE) using a Sequential Quadratic Programming (SQPLab) method which takes into account derivative information [3].

Once the Kriging surrogate model has been constructed, the maximum of the expected improvement is calculated using the DIviding RECTangles (DIRECT) algorithm [17]. At the point in the parameter space where the expected improvement is maximal, a new sample point is added. Subsequently, the value of the cost function $f$ at this new sample point is evaluated with the simulation code. When this new sample has been calculated, a new Kriging surrogate model is constructed and the process is repeated. The Kriging surrogate model is thus only used to calculate the expected improvement and thus to determine the location of the following sample point in the parameter space.

As the cost function has been defined as the difference between the forward simulation and a measurement, the values of the parameters for which $f$ is close to zero have to be determined. The optimization is halted when a sample point with a cost function value $f$ lower than the optimization tolerance $\epsilon$ has been calculated by the simulation code (or when 100 samples have been calculated).

\section{Simulation code}

In this section, the physical models used by the simulation code are described. The boundary conditions for the artery with the aneurysm are provided by an inlet model (Section 3.1) and an outlet model (Section 3.2). The artery itself is first modelled using a lumped parameter zero-dimensional model (Section 3.3), resulting in fast forward simulations to allow for extensive validation 


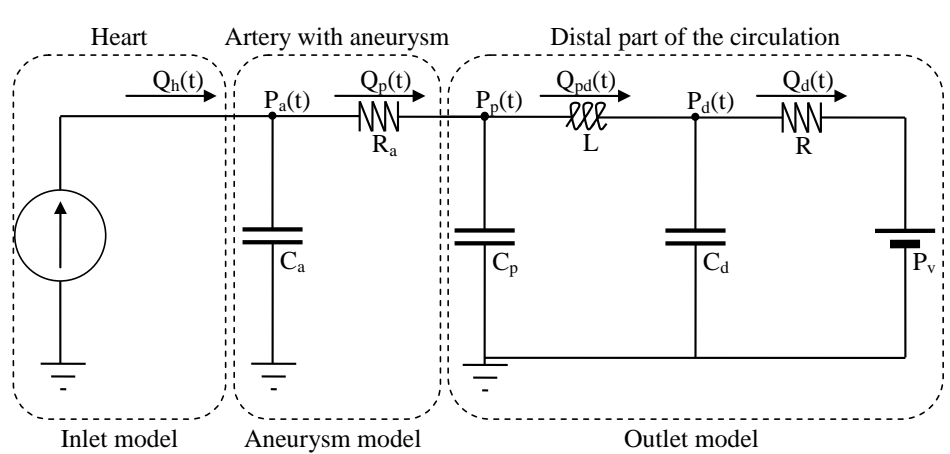

Fig. 3: Schematic of the inlet model, the zero-dimensional model of the artery with the aneurysm and the outlet model. In a second step, the zero-dimensional model of the artery with the aneurysm is replaced by a three-dimensional model.

of the parameter identification strategy and analysis of the influence of its parameters. The complete model is depicted in Figure 3. In a second step, this zero-dimensional model is replaced by a detailled three-dimensional model (Section 3.4). The parameter identification studies with the zero-dimensional model and with the three-dimensional model are thus completely independent.

\subsection{Inlet model}

At the inlet of the artery, the blood flow rate $Q_{h}$ coming from the heart is prescribed as a periodic function of the time $t$ [25]. Therefore, the time since the beginning of the current heartbeat is defined as $\tilde{t}=\bmod \left(t, T_{b}\right)$, with $\bmod$ indicating the modulo operation and $T_{b}$ the period of one heartbeat. With this definition, the blood flow rate is given by

$$
Q_{h}(t)= \begin{cases}A \tilde{t}^{2}+B \tilde{t} & : \tilde{t}<T_{s} \\ 0 & : \tilde{t} \geq T_{s} .\end{cases}
$$

The parameters $A$ and $B$ are calculated from the stroke volume $S V$ and the duration of the systole $T_{s}$, which is approximated as $\sqrt{T_{b}} / 3$.

$$
A=-\frac{6 S V}{T_{s}^{3}} \quad \text { and } \quad B=\frac{6 S V}{T_{s}^{2}} .
$$

An example of this function for two periods is displayed in Figure 4.

\subsection{Outlet model}

At the outlet of the artery, a modified windkessel model (Figure 3) determines the relation between the pressure and the blood flow rate. This model divides 


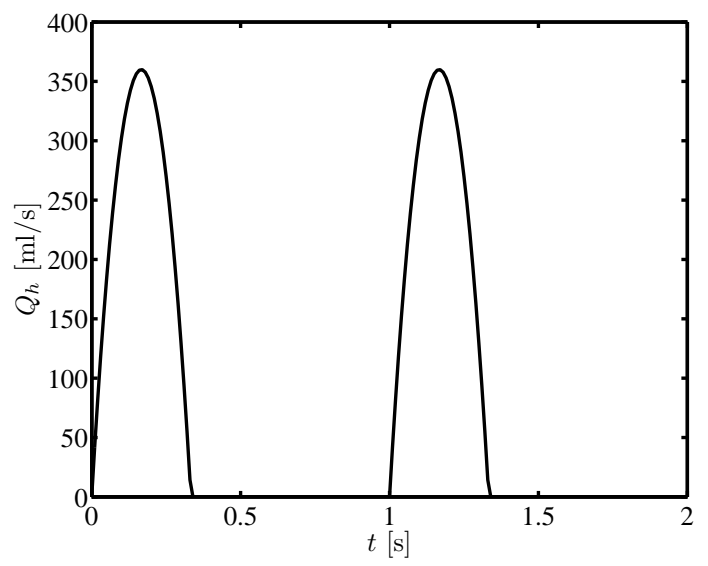

Fig. 4: The blood flow rate $Q_{h}$ coming from the inlet model as a function of time $t$ for $S V=8 \cdot 10^{-5} \mathrm{~m}^{3}$ and $T_{b}=1 \mathrm{~s}$.

the arterial tree into a proximal region (subscript $p$ ) with the arteries close to the heart and a distal region (subscript $d$ ) with the arteries further away as the arterial properties differ between those regions. The compliance of the large proximal arteries is referred to as $C_{p}$, while the compliance of the distal arteries is named $C_{d}$. The inductor $L$ represents the inertia of the blood flow. Finally, $R$ and $P_{v}$ are the peripheral resistance and the mean venous pressure, respectively $[27,25]$.

Using Kirchhoff's voltage and current laws, this modified windkessel model is characterized as

$$
\begin{aligned}
& C_{p} \frac{\mathrm{d} P_{p}(t)}{\mathrm{d} t}=Q_{p}(t)-Q_{p d}(t) \\
& C_{d} \frac{\mathrm{d} P_{d}(t)}{\mathrm{d} t}=Q_{p d}(t)-\frac{P_{d}(t)-P_{v}}{R} \\
& L \frac{\mathrm{d} Q_{p d}(t)}{\mathrm{d} t}=P_{p}(t)-P_{d}(t) .
\end{aligned}
$$

These equations are discretized in time with the first-order backward Euler scheme. The resulting linear equations

$$
\begin{aligned}
\frac{C_{p}}{\Delta t} P_{p}^{n+1}+Q_{p d}^{n+1} & =\frac{C_{p}}{\Delta t} P_{p}^{n}+Q_{p}^{n+1} \\
\left(\frac{C_{d}}{\Delta t}+\frac{1}{R}\right) P_{d}^{n+1}-Q_{p d}^{n+1} & =\frac{C_{d}}{\Delta t} P_{d}^{n}+\frac{P_{v}}{R} \\
-P_{p}^{n+1}+P_{d}^{n+1}+\frac{L}{\Delta t} Q_{p d}^{n+1} & =\frac{L}{\Delta t} Q_{p d}^{n}
\end{aligned}
$$

can be solved in every time step, yielding $P_{p}^{n+1}$ if $Q_{p}^{n+1}$ is known. The superscript $n$ indicates the time $t=n \Delta t$ with $\Delta t$ the time step size. The variables $P_{p}, P_{d}$ and $Q_{p d}$ are all initialized to zero. 


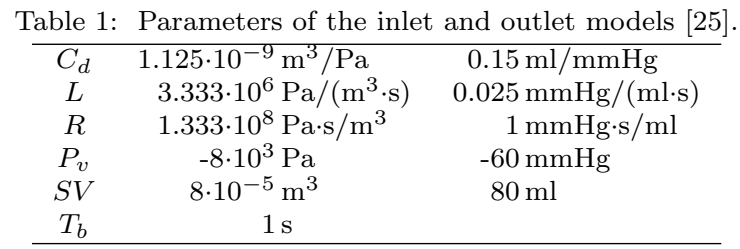

The distention of the artery with the aneurysm is significantly influenced by the pressure at its outlet. In turn, this pressure depends on the compliance of the proximal and distal arteries. Therefore, the compliance $C_{p}$ is determined by the first stiffness parameter $x_{1}$. As $x_{1}$ increases from -1 to 1 , the value of $C_{p}$ decreases from 2 to $1 / 2$ times its nominal value of $1.45 \mathrm{ml} / \mathrm{mmHg}$ according to

$$
C_{p}=\frac{1.45}{\frac{5}{4}+\frac{3}{4} x_{1}} .
$$

All other parameters of the inlet and outlet models are listed in Table 1. The mean venous pressure $P_{v}$ is manually adjusted so that $P_{p}(t)$ is positive throughout the simulation but with a minimal value close to zero since a linear elastic constitutive equation is applied. Consequently, all pressures in the simulation are relative to the minimal pressure in a heartbeat. It is thus also assumed that the initial, stressless geometry corresponds with this minimal pressure. In this case, the manual adjustment of $P_{v}$ results in a negative value.

\subsection{Zero-dimensional aneurysm model}

The zero-dimensional model of the artery with the aneurysm consists of a compliance $C_{a}$ in combination with a resistance $R_{a}$ (see Figure 3). It is characterized by

$$
C_{a} \frac{\mathrm{d} P_{a}(t)}{\mathrm{d} t}=Q_{h}(t)-\frac{P_{a}(t)-P_{p}(t)}{R_{a}},
$$

which becomes

$$
\left(\frac{C_{a}}{\Delta t}+\frac{1}{R_{a}}\right) P_{a}^{n+1}=\frac{C_{a}}{\Delta t} P_{a}^{n}+Q_{h}^{n+1}+\frac{P_{p}^{n+1}}{R_{a}}
$$

after time discretization. This lumped parameter model is coupled with the inlet model through $Q_{h}$ and to the outlet model through $P_{p}$. In a forward simulation with this zero-dimensional aneurysm model, Equations (7) and Equation (10) are combined into a linear system in the unknowns $P_{a}^{n+1}, P_{p}^{n+1}$, $P_{d}^{n+1}$ and $Q_{p d}^{n+1}$. As $Q_{h}^{n+1}$ can simply be calculated from Equation (4), it is not treated as an unknown.

The nominal values of $R_{a}$ and $C_{a}$ are calculated by assuming the artery is a straight tube with a length $\ell$ and a uniform circular cross section $a=\pi r_{o}^{2}$ 
Table 2: Parameters of the zero-dimensional and three-dimensional model of the artery with the aneurysm.

\begin{tabular}{cccc}
\hline$r_{o}$ & $0.005 \mathrm{~m}$ & $h$ & $0.003 \mathrm{~m}$ \\
$\ell$ & $0.06 \mathrm{~m}$ & $E$ & $7.5 \cdot 10^{5} \mathrm{~Pa}$ \\
$\mu_{f}$ & $0.003 \mathrm{~Pa} \cdot \mathrm{s}$ & $\nu$ & 0.45 \\
$\rho_{f}$ & $10^{3} \mathrm{~kg} / \mathrm{m}^{3}$ & $\rho_{s}$ & $1.2 \cdot 10^{3} \mathrm{~kg} / \mathrm{m}^{3}$ \\
\hline
\end{tabular}

with $r_{o}$ the initial radius. The resistance $R_{a}$ is then obtained from Poiseuille's equation

$$
R_{a}=\frac{8 \mu_{f} \ell}{\pi r_{o}^{4}}
$$

with $\mu_{f}$ the dynamic viscosity of blood. The compliance $C_{a}$ is defined as

$$
C_{a}=\frac{V}{\rho_{f} c^{2}},
$$

with $V$ the volume of the artery, $\rho_{f}$ the density of blood and $c$ the wave speed. Using the Moens-Korteweg approximation, the pulse wave speed is given by

$$
c=\sqrt{\frac{E h}{2 \rho_{f} r_{o}}},
$$

with $E$ the Young's modulus of the arterial wall and $h$ its thickness. Using the values listed in Table 2, the nominal values of $R_{a}$ and $C_{a}$ are respectively $4.125 \cdot 10^{-11} \mathrm{~Pa} \cdot \mathrm{s} / \mathrm{m}^{3}=0.0055 \mathrm{mmHg} \cdot \mathrm{s} / \mathrm{ml}$ and $3.733 \cdot 10^{5} \mathrm{~m}^{3} / \mathrm{Pa}=0.0028 \mathrm{ml} / \mathrm{mmHg}$. The wave speed $c$ is approximately $15 \mathrm{~m} / \mathrm{s}$, which is rather high.

The distention of the artery depends on its compliance, so $C_{a}$ is modified using the second stiffness parameter $x_{2}$. This is achieved by multiplying the nominal value of $E$ with a factor containing $x_{2}$

$$
E=750000\left(1+\frac{1}{3} x_{2}\right) .
$$

As $x_{2}$ increases from -1 to 1 , the value of $C_{a}$ thus decreases from $3 / 2$ to $3 / 4$ times its nominal value.

The initial value of the artery's inner radius is listed in Table 2. After each time step, this radius is calculated from the artery's volume

$$
r^{n+1}=\sqrt{\frac{V^{n+1}}{\pi \ell}}
$$

which is in turn obtained by integrating the net blood inflow

$$
V^{n+1}=V^{n}+\left(Q_{h}^{n+1}-Q_{p}^{n+1}\right) \Delta t .
$$

For given inputs $x_{1}$ and $x_{2}$, the output of this aneurysm model combined with the inlet and outlet models is the value of $r$ in all time steps. Each forward 


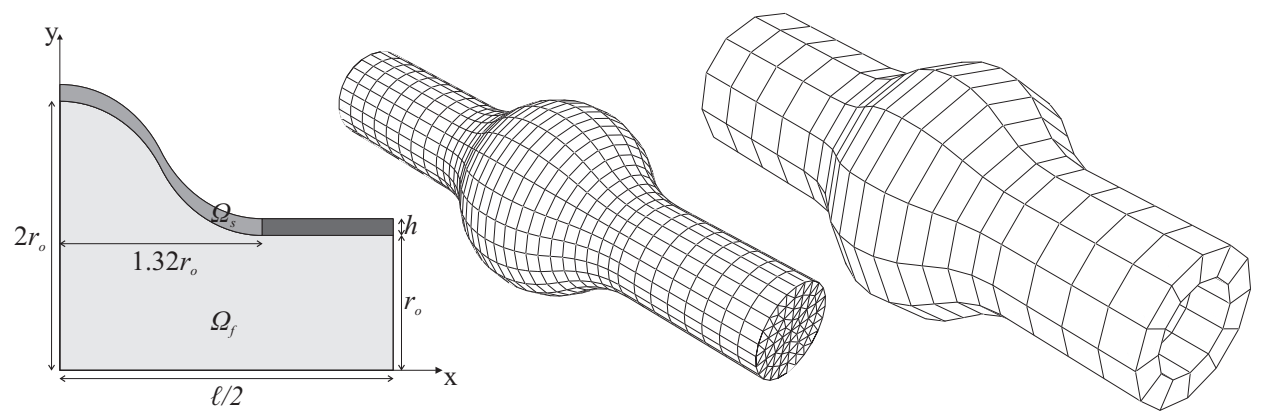

Fig. 5: Schematic of the geometry (left), together with the grid in the fluid (middle) and structure (right) domain in the three-dimensional model of the artery with the aneurysm. The geometry is axisymmetric around the $\mathrm{x}$-axis and symmetric around a plane through the origin with the $\mathrm{x}$-axis as normal.

simulation continues for 10 heartbeats with a time step $\Delta t=0.01 \mathrm{~s}$. This time step size has been selected based on a time step convergence study.

To mimic the measurement from an imaging technique, a reference simulation is performed with $x_{1}^{*}$ and $x_{2}^{*}$, yielding $r^{*}$ in all time steps. Of course, the values of $x_{1}^{*}$ and $x_{2}^{*}$ are further assumed unknown as they have to be determined by the inverse modelling. The cost function is finally defined as

$$
f_{0 D}\left(x_{1}, x_{2}\right)=\frac{\Delta t}{T_{b}} \frac{\sum_{n}\left|r^{n}\left(x_{1}, x_{2}\right)-r^{n *}\right|}{\max _{n}\left(r^{n *}\right)-\min _{n}\left(r^{n *}\right)} .
$$

In the above equation, the index $n$ is limited to the last heartbeat of the simulation.

\subsection{Three-dimensional aneurysm model}

In the three-dimensional model, the artery with the aneurysm is divided into a fluid domain $\Omega_{f}$ and a structure domain $\Omega_{s}$ (Figure 5). Initially, the radius at the inlet and outlet is $r_{o}$, the radius at the aneurysm is $2 r_{o}$. This simplified geometry is axisymmetric around the x-axis and symmetric around a plane through the origin with the x-axis as normal. The boundaries of the fluid and structure domain are indicated as $\Gamma_{f}$ and $\Gamma_{s}$, respectively. The fluid-structure interface $\Gamma_{i}=\Gamma_{f} \cap \Gamma_{s}$ is the common boundary of these domains.

The unsteady blood flow is governed by the conservation of mass and the Navier-Stokes equations, given by

$$
\frac{\partial \rho_{f}}{\partial t}+\nabla \cdot\left(\rho_{f} \mathbf{v}\right)=0
$$




$$
\frac{\partial \rho_{f} \mathbf{v}}{\partial t}+\nabla \cdot\left(\rho_{f} \mathbf{v v}\right)-\nabla \cdot \bar{\sigma}_{f}=\mathbf{0}
$$

for each point in $\Omega_{f}$. In these equations, $\rho_{f}$ is the blood density and $\mathbf{v}$ the flow velocity. Blood is modelled as an incompressible, Newtonian fluid with dynamic viscosity $\mu_{f}$, so the stress tensor $\bar{\sigma}_{f}$ is defined as

$$
\bar{\sigma}_{f}=-p \bar{I}+2 \mu_{f} \bar{\epsilon}_{f}
$$

with $p$ the pressure and $\bar{I}$ the unit tensor. The rate of strain tensor $\bar{\epsilon}_{f}$ is given by

$$
\bar{\epsilon}_{f}=\frac{1}{2}\left[\nabla \mathbf{v}+(\nabla \mathbf{v})^{\mathrm{T}}\right] .
$$

The deformation $\mathbf{u}$ of the arterial wall is determined by the conservation of momentum

$$
\rho_{s} \frac{\mathrm{d}^{2} \mathbf{u}}{\mathrm{d} t^{2}}-\nabla \cdot \bar{\sigma}_{s}=\mathbf{0}
$$

for each point in $\Omega_{s}$ with $\rho_{s}$ the density of the arterial wall and $\bar{\sigma}_{s}$ the Cauchy stress tensor. In these large displacement calculations, the relation between the second Piola-Kirchhoff stress tensor $\bar{S}_{s}$ and the Green-Lagrange strain tensor $\bar{E}_{s}$ is imposed by the constitutive equation of the material. The second Piola-Kirchhoff stress tensor combines forces in the reference configuration with areas in the reference configuration, whereas the Cauchy stress tensor combines forces in the deformed configuration with areas in the deformed configuration. The relation between these tensors is given by

$$
\bar{S}=J \bar{F}^{-1} \bar{\sigma}_{s} \bar{F}^{-\mathrm{T}}
$$

with $\bar{F}$ the deformation gradient tensor and $J=\operatorname{det}(\bar{F})$. The Green-Lagrange strain tensor for large displacements is given by

$$
\bar{E}_{s}=\frac{1}{2}\left[\nabla \mathbf{u}+(\nabla \mathbf{u})^{\mathrm{T}}+(\nabla \mathbf{u})^{\mathrm{T}} \nabla \mathbf{u}\right] .
$$

All displacements are relative to the initial (reference) geometry.

The equilibrium conditions on the fluid-structure interface are the kinematic condition

$$
\mathbf{v}=\frac{\mathrm{d} \mathbf{u}}{\mathrm{d} t}
$$

and the dynamic condition

$$
\bar{\sigma}_{f} \cdot \mathbf{n}_{f}=-\bar{\sigma}_{s} \cdot \mathbf{n}_{s},
$$

which stipulate that the velocity and the stress have to be the same on both sides of the interface. The vector $\mathbf{n}_{f, s}$ is the unit normal that points outwards from the domain $\Omega_{f, s}$. A Dirichlet-Neumann decomposition of the fluidstructure interaction problem is applied, so the flow equations are solved with a given displacement of the fluid-structure interface and the structural equations are solved with a given stress on the interface. 
The flow equations (Equations (18)) and the structural equations (Equation (20)) are solved by coupling a flow solver (Fluent 12.1, Ansys Inc.) with a structural solver (Abaqus 6.7, Dassault Systèmes). This is the so-called partitioned approach to the simulation of fluid-structure interaction. In every time step, coupling iterations are performed between these solvers to enforce the equilibrium conditions (Equations (23)). A simple scheme for these coupling iterations would be to solve the flow equations for a given interface displacement, followed by solving the structural equations using the resulting stress on the interface and finally giving the resulting interface displacement back to the flow solver. However, this so-called Gauss-Seidel scheme does not converge for this case, which is well understood $[4,10,8]$. Instead, the Interface Quasi-Newton algorithm with an approximation for the Inverse of the Jacobian from a Least-Squares model (IQN-ILS) has been applied [9,11]. This coupling scheme uses the displacement and stress on the fluid-structure interface during the coupling iterations to accelerate the convergence of these iterations. Moreover, it treats both solvers as black boxes.

At the inlet of the fluid domain, the blood flow rate $Q_{h}$ from the inlet model is imposed. The pressure level in the incompressible fluid is only known up to an arbitrary constant which is fixed by applying a zero-pressure boundary condition at the outlet. After each flow calculation, the blood flow rate $Q_{p}$ at the outlet is calculated and given to the outlet model which then determines $P_{p}$. The physically correct pressure level is then obtained by adding $P_{p}$ to the pressure in the entire fluid domain. The structure is clamped at both ends in the axial and circumferential direction. Both the fluid and the structure domain are initially at rest and stressless.

The finite volume flow solver solves the Navier-Stokes equations in Arbitrary Lagrangian-Eulerian (ALE) formulation using the Pressure-Implicit with Splitting of Operators (PISO) pressure-velocity coupling. The time discretization is first-order backward Euler. The momentum equations are discretized in space using the second-order upwind scheme, while the pressure is interpolated from the cells to the faces using momentum equation coefficients. The grid throughout the fluid domain is adapted to the displacement of the fluidstructure interface by replacing the cell edges with springs. The finite element structural solver uses implicit Hilber-Hughes-Taylor time integration with a numerical dissipation factor of $\alpha_{s}=-0.15$. It takes into account the geometric nonlinearities due to the large deformation of the structure. However, the deformations are in the order of $5 \%$, so small deformation stress-strain measures would have been sufficient. The constitutive equation for the structure is a linear elastic material law with Young's modulus $E$ and Poisson's coefficient $\nu$.

All parameters have the same value as for the zero-dimensional model (Table 2). The fluid grid consists of 4160 triangular prisms and the structural grid contains 240 brick elements with 8 nodes. The time step is $\Delta t=0.005 \mathrm{~s}$, which is divided into smaller increments in the structural solver.

As in Equation (14) for the zero-dimensional model, the stiffness of the aneurysm is modified by multiplying the nominal value of $E$ with the same 

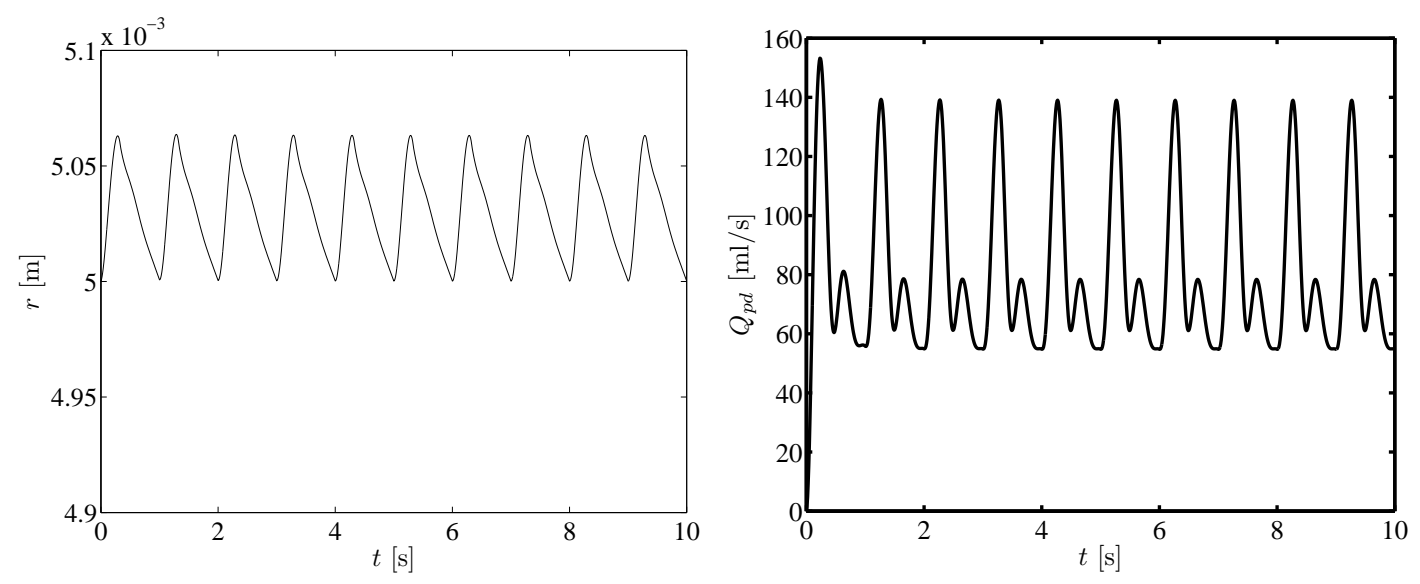

Fig. 6: The radius $r$ (left) and blood flow rate $Q_{p d}$ (right) as a function of time $t$ in a simulation with the zero-dimensional aneurysm model and nominal parameter values $\left(x_{1}=\right.$ $\left.x_{2}=0\right)$.

factor containing $x_{2}$. However, the stiffness is only modified in the light grey part of the structure as depicted in Figure 5. The cost function is also identical to the one for the zero-dimensional model in Equation (17). The radius $r$ is measured at the intersection between the y-axis and the fluid-structure interface.

\section{Results}

\subsection{Zero-dimensional aneurysm model}

The inlet and outlet models are first coupled to the zero-dimensional aneurysm model. The radius $r$ and blood flow rate $Q_{p d}$ as a function of time $t$ for nominal parameter values $\left(x_{1}=x_{2}=0\right)$ are depicted in Figure 6 . It can be observed that the distention and flow rate become periodic after a few heartbeats; the same is true for the other variables.

The dependence of the total number of samples to reach the optimum on the location of the optimum in the design space is depicted in Figure 7. The optimization has been performed for 121 positions of the optimum, uniformly distributed in the design space. Therefore, $x_{1}^{*}$ and $x_{2}^{*}$ are increased independently from -1 to 1 in steps of 0.2 . For each position of the optimum, a parameter identification has been performed and the number of samples has been counted. Each of these calculations has been performed with the same 11 initial samples. If $x_{1}^{*}$ and $x_{2}^{*}$ are equal to \pm 1 , then the criterion $f_{0 D}<\epsilon$ is satisfied immediately once all initial samples have been calculated as the corners of the design space are included in the set of initial samples. The max- 

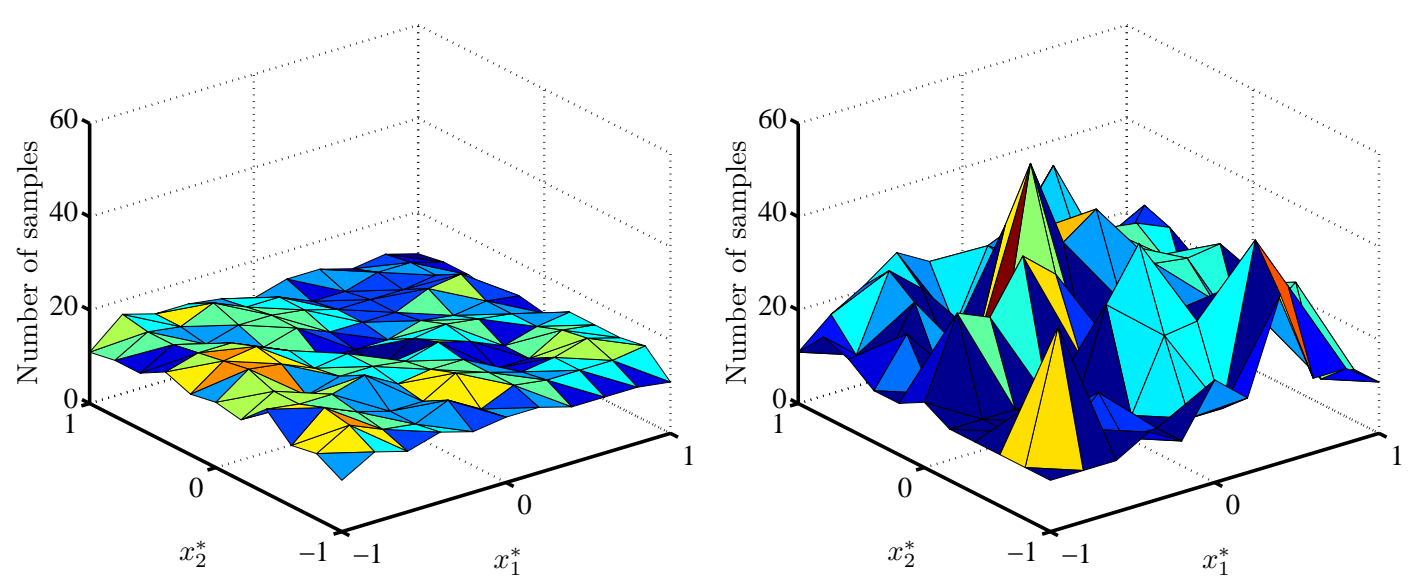

Fig. 7: The total number of samples to reach the optimum as a function of the location of the optimum for tolerance $\epsilon=1 \%$ (left) and $0.1 \%$ (right). All these optimizations have been performed with 11 initial samples and the zero-dimensional model.

imal difference in total number of samples throughout the design space is 11 for $\epsilon=1 \%$ and 43 for $\epsilon=0.1 \%$, so decreasing the optimization tolerance increases the dependence of the total number of samples on the location of the optimum. Decreasing the optimization tolerance $\epsilon$ from $1 \%$ (Figure 7a) to $0.1 \%$ (Figure 7b) approximately causes an increase in the number of samples (averaged over all values of $x_{1}^{*}$ and $x_{2}^{*}$ ) from 14.5 to 19.6.

Figure 8 shows the influence of the number initial samples on the total number of samples before the criterion $f_{0 D}<\epsilon$ is reached. For each combination of $\epsilon$ and the number of initial samples, the optimization has been performed for 121 positions of the optimum, uniformly distributed in the design space. Therefore, $x_{1}^{*}$ and $x_{2}^{*}$ are again increased independently from -1 to 1 in steps of 0.2 . If at least 11 initial samples are generated, the optimizer finds the minimum of $f_{0 D}$ after about 2 or 3 samples once the evaluation of the initial samples is complete if $\epsilon=1 \%$ and after about 10 samples if $\epsilon=0.1 \%$. If fewer than 11 initial samples are used, the total number of samples remains more or less constant. The standard deviation increases significantly as the optimization tolerance $\epsilon$ is reduced from $1 \%$ to $0.1 \%$, which means that the total number of samples becomes more sensitive to the location of the optimum. This confirms the information obtained from Figure 7.

As mentioned before, the initial set of samples is generated by a Latin hypercube design, together with the corner points of the parameter space. Because the Latin hypercube design can contain one of the corners of the design space and duplicate samples are removed, not every number of initial samples in the range 4 to 24 can be realized. Consequently, the total number of samples cannot be shown for each number of initial samples in that range. 

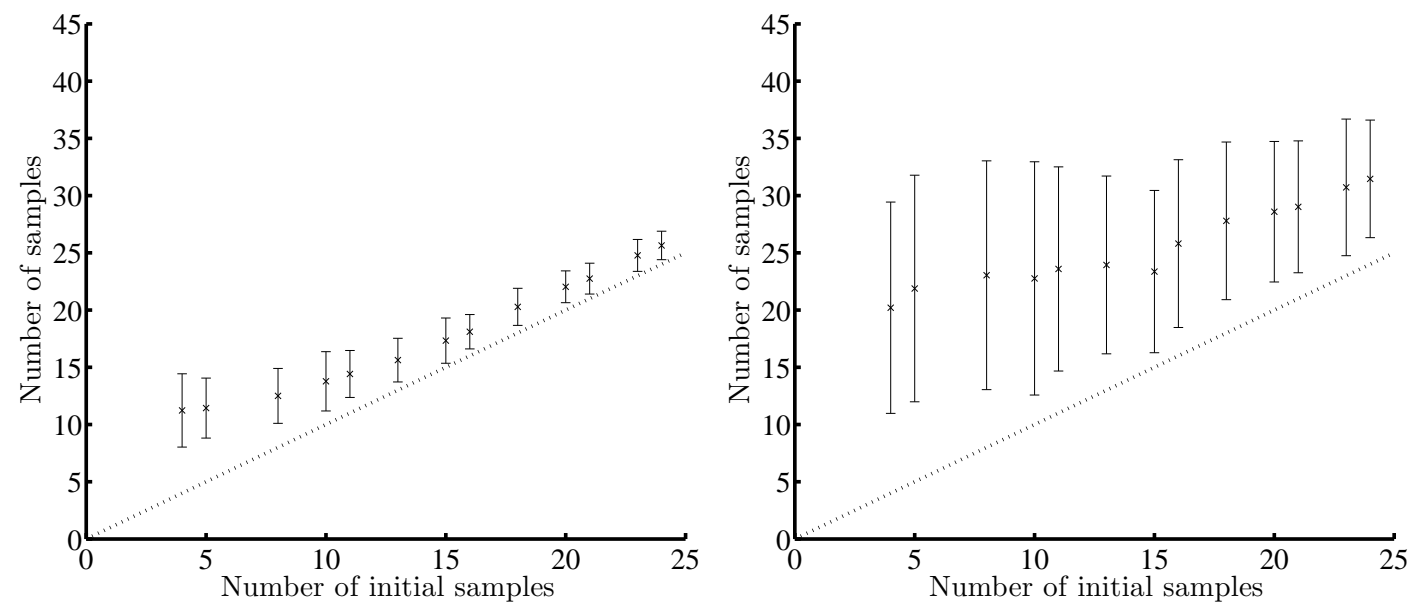

Fig. 8: The total number of samples to reach the optimum as a function of the number of initial samples for tolerance $\epsilon=1 \%$ (left) and $0.1 \%$ (right). For each combination of $\epsilon$ and the number of initial samples, the optimization has been performed for a range of $x_{1}^{*}$ and $x_{2}^{*}$. The mean \pm standard deviation from all these optimizations with the zero-dimensional model is depicted.

The difference between $x_{i}^{*}$ and $x_{i}(i=1,2)$ during a representative optimization is shown in Figure 9a. In this optimization, there were 11 initial samples, $\epsilon=1 \%, x_{1}^{*}=-0.2$ and $x_{2}^{*}=-0.4$. The evolution of $f_{0 D}$ during the same optimization is depicted in Figure 9b. It can be observed that the convergence is not monotonic as the surrogate-based optimization balances between exploration and exploitation. Nevertheless, the difference between $x_{i}^{*}$ and $x_{i}$ is small when $f_{0 D}$ is small, which gives confidence for situations where $x_{i}^{*}$ is really unknown.

Figure 9a shows that the error on the parameters is smaller than $10^{-2}$ at convergence. To identify the parameters with a similar accuracy using uniform sampling of the parameter space, a step size no larger than $10^{-2}$ should be applied. If the parameter space Equation (3) were uniformly sampled with this step size, then 40000 calculations with the simulation code would have to be performed. By contrast, the surrogate model only requires approximately 50 calculations. Moreover, the computational cost of the surrogate model itself is low compared to the simulation code. As a result, the surrogate model drastically reduces the computational cost compared to uniform sampling.

The convergence path for this optimization can be seen in Figure 10a. Only the additional samples (i.e. the samples added after the initial samples) are indicated. It can be observed that the optimization algorithm has not only added a cluster of samples in the neighbourhoud of the optimum but also individual samples in other parts of the design space. Figure 10b shows the final Kriging model that is constructed with all samples. The coloured surface 

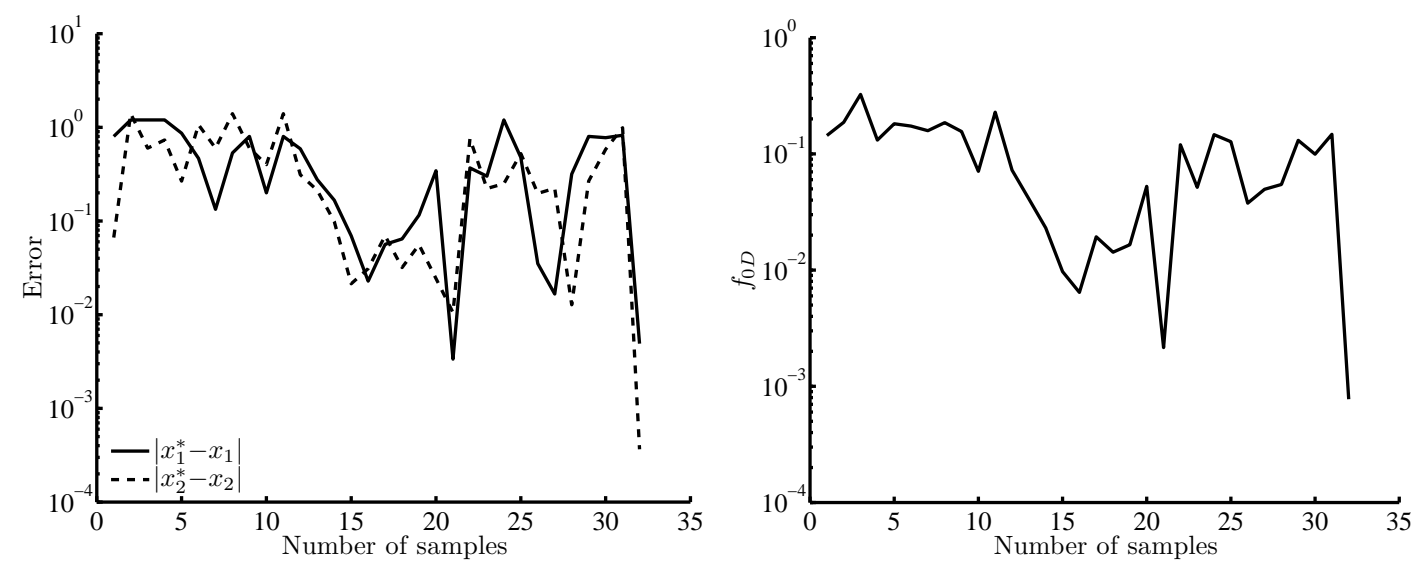

Fig. 9: The evolution of $\left|x_{i}^{*}-x_{i}\right|$ (left) and $f_{0 D}$ (right) in an optimization with the zerodimensional model using 11 initial samples, $\epsilon=1 \%, x_{1}^{*}=-0.2$ and $x_{2}^{*}=-0.4$.

in this figure is the surrogate model. Despite the non-uniform distribution of the samples, this surrogate model does not display wiggles or peaks in between the samples. This figure also shows that the maximal cost function value is approximately 0.3 , which means that the difference between the simulation and the measurement is up to $30 \%$. This percentage is an average over all time steps and with respect to the maximal displacement in the measurement.

\subsection{Three-dimensional aneurysm model}

As the results in the previous section became periodic quickly, only 3 heartbeats are simulated with the three-dimensional model. Also, 11 initial samples are used as this resulted in a low total number of samples with the zero-dimensional model. The optimum that needs to be found is located at $x_{1}^{*}=-0.2$ and $x_{2}^{*}=-0.4$. The radius $r$ and blood flow rate $Q_{p d}$ as a function of time $t$ at the optimum are depicted in Figure 11.

Each forward simulation takes approximately 6 hours on 3 cores of an Intel Xeon X5355 2.66GHz processor. In each time step, on average 5.13 coupling iterations per time step are required to reach the convergence criterion of the fluid-structure interaction coupling iterations. Figure 12 depicts velocity vectors in the fluid and contours of the von Mises stress in the structure at three different instants in the last period for $x_{1}^{*}=-0.2$ and $x_{2}^{*}=-0.4$.

The difference between $x_{i}^{*}$ and $x_{i}(i=1,2)$ is shown in Figure 13, together with the evolution of $f_{3 D}$ during the optimization. It can again be observed that the convergence is not monotonic but that $\left|x_{i}^{*}-x_{i}\right|$ is small when $f_{3 D}$ is small. The convergence tolerance $\epsilon=1 \%$ is reached after 15 samples, while 42 

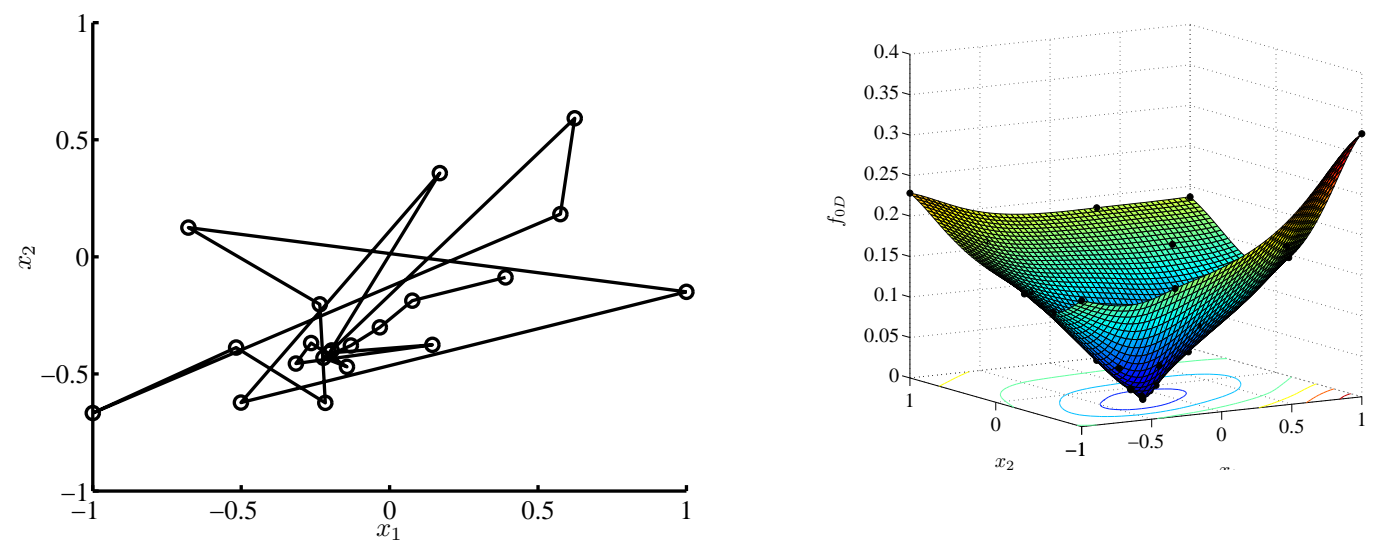

Fig. 10: The location of the additional samples (left) and the final Kriging surrogate model (right) in an optimization with the zero-dimensional model using 11 initial samples, $\epsilon=1 \%$, $x_{1}^{*}=-0.2$ and $x_{2}^{*}=-0.4$. The samples are indicated by black dots.
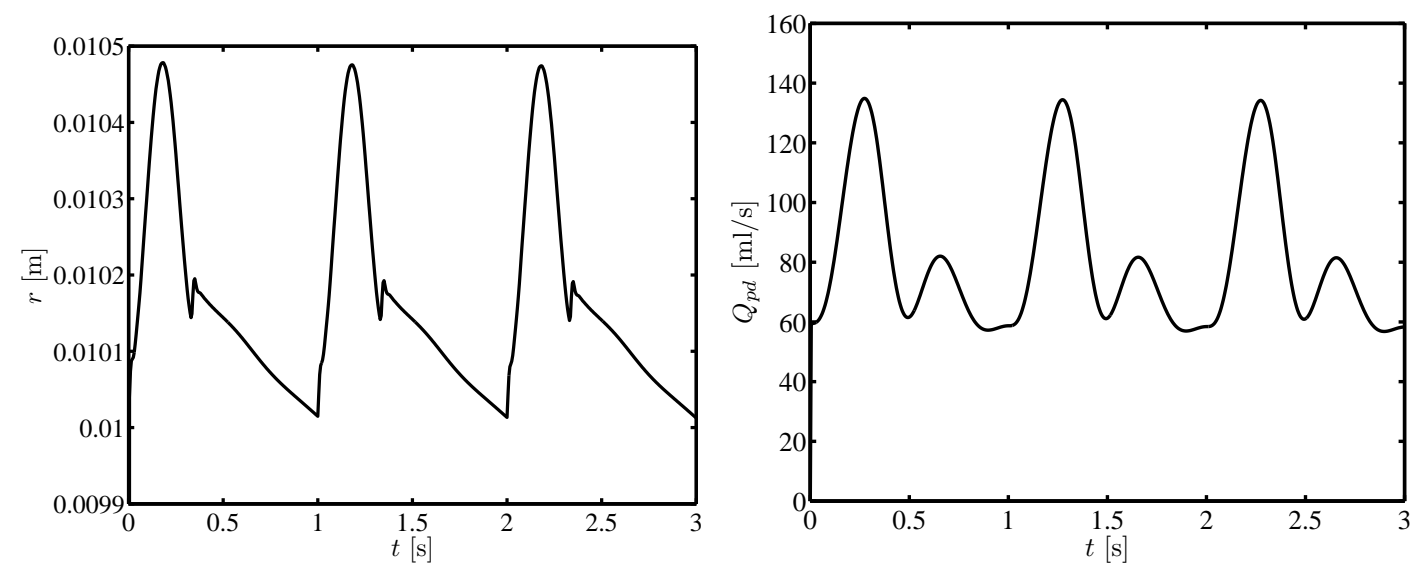

Fig. 11: The radius $r$ (left) and blood flow rate $Q_{p d}$ (right) as a function of time $t$ in a simulation with the three-dimensional aneurysm model and parameter values $x_{1}^{*}=-0.2$ and $x_{2}^{*}=-0.4$. 


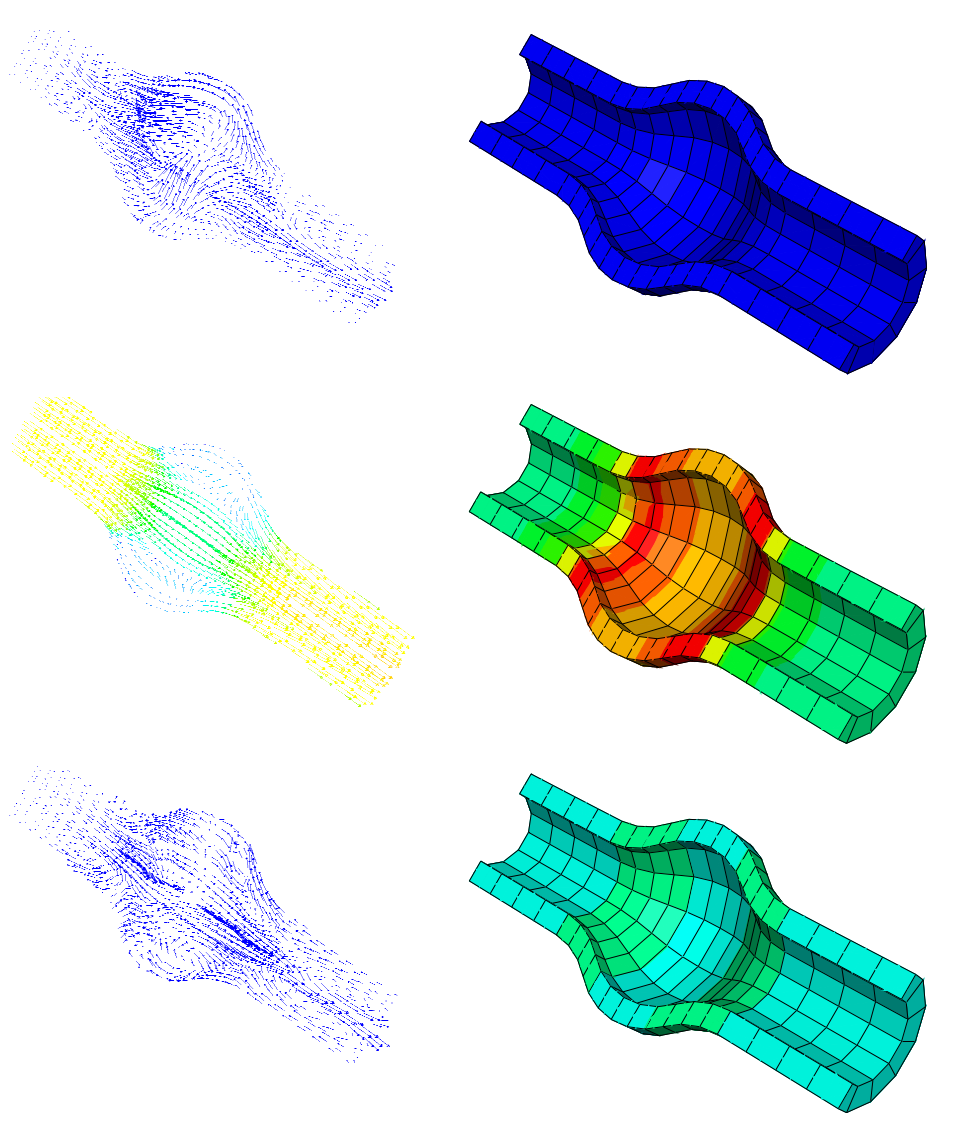

Fig. 12: Velocity vectors in the fluid and contours of the von Mises stress in the structure at $2.00 \mathrm{~s}, 2.25 \mathrm{~s}$ and $2.50 \mathrm{~s}$ in a simulation with the three-dimensional model and parameter values $x_{1}^{*}=-0.2$ and $x_{2}^{*}=-0.4$. The velocity range is 0 (blue) to $5 \mathrm{~m} / \mathrm{s}$ (red) and the stress range is $5 \cdot 10^{2} \mathrm{~Pa}$ (blue) to $25 \cdot 10^{3} \mathrm{~Pa}$ (red).

samples are required to reach the tolerance $\epsilon=0.1 \%$. The minimum of $f_{3 D}$ over all samples is $8.92 \cdot 10^{-4}$.

The convergence path for this optimization can be seen in Figure 14a, with only the additional samples indicated. The optimization algorithm has positioned most additional samples near the optimum and the distance between the samples increases further away from the optimum. Figure 14b shows the final Kriging model that is constructed with all samples. Again, this Kriging surrogate model is smooth in between the samples, despite the non-uniform distribution of the samples. For the 3D model, the maximal cost function value is approximately 0.2 , which signifies a difference of up to $20 \%$ between the simulation and the measurement. Despite the nonlinearities in the physics, the relation between the parameters and the cost function has a cone shape, which is straightforward to optimize. 

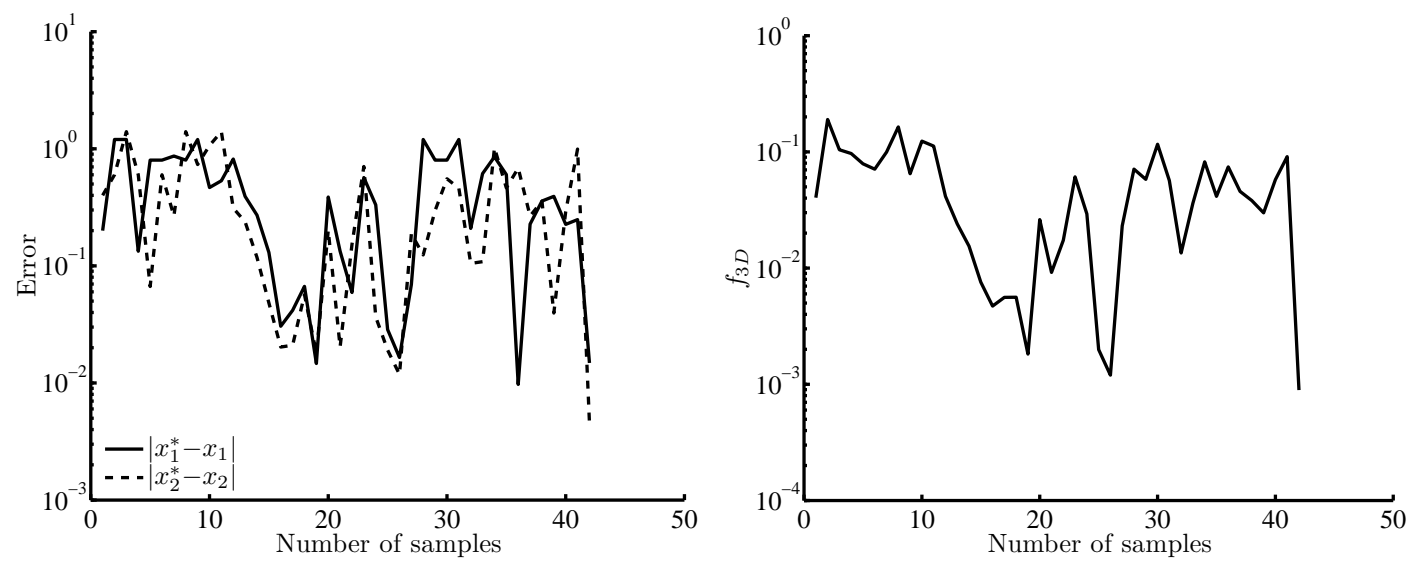

Fig. 13: The evolution of $\left|x_{i}^{*}-x_{i}\right|$ (left) and $f_{3 D}$ (right) in an optimization with the three-dimensional model using 11 initial samples, $x_{1}^{*}=-0.2$ and $x_{2}^{*}=-0.4$.
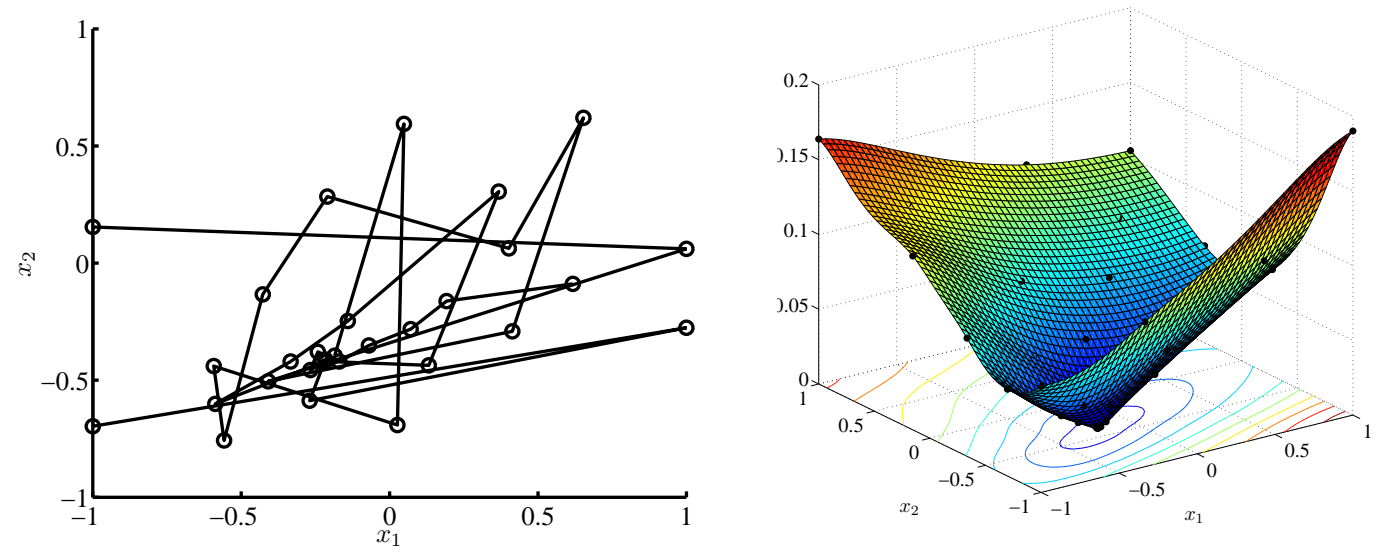

Fig. 14: The location of the additional samples (left) and the Kriging surrogate model after 42 samples (right) in the optimization with the three-dimensional model using 11 initial samples, $x_{1}^{*}=-0.2$ and $x_{2}^{*}=-0.4$. The samples are indicated by black dots.

\section{Conclusions}

The increase in computer power and the use of surrogate-based optimization make it feasible to identify an aneurysm's stiffness using a three-dimensional FSI simulation. Both the coupling algorithm for the three-dimensional partitioned fluid-structure interaction simulations and the algorithm for the surrogate- 
based optimization treat the flow solver and structural solver as black boxes. The influence of the parameters in the surrogate-based optimization has first been analyzed using a zero-dimensional model.

However, the geometry and models in this work are simplified. It would also be very expensive to identify a large number of parameters. Future work will be to use patient-specific data for the geometry, input model and output model. Subsequently, real measurements instead of synthetic measurement data from a simulation will be incorporated, as the applied surrogate-based optimization can cope with noisy data if a suitable surrogate model is used [31].

Acknowledgements Joris Degroote is funded by a postdoctoral fellowship of the Research Foundation - Flanders (FWO). Ivo Couckuyt is funded by the Institute for the Promotion of Innovation through Science and Technology in Flanders (IWT-Vlaanderen).

\section{References}

1. Alexandrov, N., Dennis, J., Lewis, R., Torczon, V.: A trust region framework for managing the use of approximation models in optimization. Structural and Multidisciplinary Optimization 15(1), 16-23 (1998)

2. Astorino, M., Gerbeau, J.F., Pantz, O., Traore, K.F.: Fluid-structure interaction and multi-body contact: Application to aortic valves. Computer Methods in Applied Mechanics and Engineering 198(45-46), 3603-3612 (2009)

3. Bonnans, J., Gilbert, J., Lemaréchal, C., Sagastizábal, C.: Numerical Optimization: Theoretical and Practical Aspects. Springer (2006)

4. Causin, P., Gerbeau, J.F., Nobile, F.: Added-mass effect in the design of partitioned algorithms for fluid-structure problems. Computer Methods in Applied Mechanics and Engineering 194(42-44), 4506-4527 (2005)

5. Couckuyt, I., Declercq, F., Dhaene, T., Rogier, H., Knockaert, L.: Surrogate-based infill optimization applied to electromagnetic problems. International Journal of RF and Microwave Computer-Aided Engineering 20(5), 492-501 (2010)

6. Cressie, N.: The origins of Kriging. Mathematical Geology 22(3), 239-252 (1990)

7. van Dam, E., Husslage, B., den Hertog, D., Melissen, H.: Maximin Latin hypercube designs in two dimensions. Operations Research 55(1), 158-169 (2007)

8. Degroote, J., Annerel, S., Vierendeels, J.: Stability analysis of Gauss-Seidel iterations in a partitioned simulation of fluid-structure interaction. Computers \& Structures $88(5-6)$, 263-270 (2010)

9. Degroote, J., Bathe, K.J., Vierendeels, J.: Performance of a new partitioned procedure versus a monolithic procedure in fluid-structure interaction. Computers \& Structures 87(11-12), 793-801 (2009)

10. Degroote, J., Bruggeman, P., Haelterman, R., Vierendeels, J.: Stability of a coupling technique for partitioned solvers in FSI applications. Computers \& Structures 86(2324), 2224-2234 (2008)

11. Degroote, J., Haelterman, R., Annerel, S., Bruggeman, P., Vierendeels, J.: Performance of partitioned procedures in fluid-structure interaction. Computers \& Structures $\mathbf{8 8}(7-$ 8), 446-457 (2010)

12. Forrester, A., Sobester, A., Keane, A.: Engineering Design via Surrogate Modelling: A Practical Guide. Wiley, New York (2008)

13. Gee, M., Förster, C., Wall, W.: A computational strategy for prestressing patient-specific biomechanical problems under finite deformation. International Journal for Numerical Methods in Biomedical Engineering 26(1), 52-72 (2010)

14. Goel, T., Haftka, R., Shyy, W., Queipo, N.: Ensemble of surrogates. Structural and Multidisciplinary Optimization 33(3), 199-216 (2007) 
15. Gorissen, D., Couckuyt, I., Demeester, P., Dhaene, T., Crombecq, K.: A surrogate modeling and adaptive sampling toolbox for computer based design. Journal of Machine Learning Research 11, 2051-2055 (2010)

16. Haftka, R.: Combining global and local approximations. American Institute of Aeronautics and Astronautics Journal 29(9), 1523-1525 (1991)

17. Jones, D., Perttunen, C., Stuckman, B.: Lipschitzian optimization without the Lipschitz constant. Journal of Optimization Theory and Applications 79(1), 157-181 (1993)

18. Jones, D., Schonlau, M., Welch, W.: Efficient global optimization of expensive black-box functions. Journal of Global Optimization 13(4), 455-492 (1998)

19. Küttler, U., Gee, M., Förster, C., Comerford, A., Wall, W.: Coupling strategies for biomedical fluid-structure interaction problems. International Journal for Numerical Methods in Biomedical Engineering 26(3-4), 305-321 (2010)

20. Lagrée, P.Y.: An inverse technique to deduce the elasticity of a large artery. The European Physical Journal - Applied Physics 9(2), 153-163 (2000)

21. Martin, V., Clément, F., Decoene, A., Gerbeau, J.F.: Parameter identification for a one-dimensional blood flow model. ESAIM: Proceedings 14, 174-200 (2005)

22. Minasny, B., McBratney, A.: The Matérn function as a general model for soil variograms Geoderma 128(3-4), 192-207 (2005)

23. Mockus, J., Tiesis, V., Zilinskas, A.: The application of Bayesian methods for seeking the extremum. In: L. Dixon, G. Szego (eds.) Towards Global Optimization, 2 edn., pp. 117-130. North-Holland, Amsterdam, the Netherlands (1978)

24. Ong, Y.S., Nair, P., Keane, A.: Evolutionary optimization of computationally expensive problems via surrogate modeling. American Institute of Aeronautics and Astronautics Journal 41(4), 687-696 (2003)

25. Parlikar, T., Heldt, T., Verghese, G.: Cycle-averaged models of cardiovascular dynamics. IEEE Transactions on Circuits and Systems-I 53(11), 2459-2468 (2006)

26. Queipo, N., Haftka, R., Shyy, W., Goel, T., Vaidyanathana, R., Tucker, P.: Surrogatebased analysis and optimization. Progress in Aerospace Sciences 41(1), 1-28 (2005)

27. Rietzschel, E., Boeykens, E., De Buyzere, M., Duprez, D., Clement, D.: A comparison between systolic and diastolic pulse contour analysis in the evaluation of arterial stiffness. Hypertension: Journal of the American Heart Association 37(6), 15-22 (2001)

28. Robinson, T., Eldred, M., Willcox, K., Haimes, R.: Surrogate-based optimization using multifidelity models with variable parameterization and corrected space mapping. American Institute of Aeronautics and Astronautics Journal 46(11), 2814-2822 (2008)

29. Roustant, O., Ginsbourger, D., Deville, Y.: Dicekriging, diceoptim: Two r packages for the analysis of computer experiments by kriging-based metamodeling and optimization. http://hal.archives-ouvertes.fr/hal-00495766 (2010)

30. Sacks, J., Welch, W., Mitchell, T., Wynn, H.: Design and analysis of computer experiments. Statistical Science 4(4), 409-435 (1989)

31. Sakata, S., Ashida, F., Zako, M.: On applying Kriging-based approximate optimization to inaccurate data. Computer Methods in Applied Mechanics and Engineering 196(1316), 2055-2069 (2007)

32. Viana, F.: Surrogates Toolbox User's Guide. Gainesville, FL, USA (2010). URL http://sites.google.com/site/felipeacviana/surrogatestoolbox

33. Zhou, Z., Ong, Y.S., Nair, P., Keane, A., Lum, K.: Combining global and local surrogate models to accelerate evolutionary optimization. IEEE Transactions on Systems, Man and Cybernetics - Part C 37(1), 66-76 (2007) 